\title{
Considerations for two inoculation methods of rabbit hepatic tumors: Pathology and image features
}

\author{
JUN-HUI SUN ${ }^{1,2}$, YUE-LIN ZHANG ${ }^{1,2}$, CHUN-HUI NIE $^{1,2}$, XIAO-BO YU ${ }^{1,2}$, \\ HAI-YANG XIE ${ }^{1,2}$, LIN ZHOU ${ }^{1,2}$ and SHU-SEN ZHENG ${ }^{1,2}$
}

\author{
${ }^{1}$ Key Laboratory of Combined Multi-Organ Transplantation, Ministry of Public Health, and \\ Key Laboratory of Organ Transplantation; ${ }^{2}$ Division of Hepatobiliary and Pancreatic Surgery, Department of Surgery, \\ First Affiliated Hospital, School of Medicine, Zhejiang University, Hangzhou, Zhejiang, P.R. China
}

Received October 15, 2011; Accepted December 12, 2011

DOI: $10.3892 / \mathrm{etm} .2011 .435$

\begin{abstract}
The rabbit VX2 hepatic tumor model has been widely used for the investigation of liver cancer. The present study was designed to compare two inoculation methods in order to identify an improved modality for establishing rabbit hepatic tumors. A total of 44 rabbits were randomly divided into 2 groups. All inoculations were performed by open laparotomy. In group 1, 22 rabbits received a VX2 tumor cell suspension in a volume of $0.2 \mathrm{ml}$, which was slowly injected into the rabbit liver parenchyma using an 18-gauge needle. In group 2, a 0.2-ml suspension of small, minced tumor fragments of a VX2 carcinoma, inserted into an 18-gauge needle, was implanted into the liver parenchyma. The tumor survival ratio, size and extrahepatic metastases were evaluated and compared using computed tomography (CT)-scan, angiography and pathological specimens. At 2 weeks following implantation, successful liver tumor growth was achieved in 14 out of the 22 rabbits in group $1(63.6 \%)$ and 21 out of the 22 rabbits in group $2(95.5 \%)(\mathrm{P}<0.05)$. Further sub-analysis revealed that single nodular tumors in the liver were observed in 4 out of the 14 rabbits in group $1(28.6 \%)$ and 14 out of the 21 rabbits in group $2(66.7 \%)$, and that multinodular tumors in the liver were observed in 10 out of the 14 rabbits in group $1(71.4 \%)$ and 7 out of the 21 rabbits in group $2(33.3 \%)(\mathrm{P}<0.05)$. Extrahepatic metastases were observed in 5 out of the 14 rabbits in group 1 $(35.7 \%)$ and 1 of the 21 rabbits in group $2(4.8 \%)(\mathrm{P}<0.05)$. Imaging findings of hepatic tumors were in concordance with histopathology. In conclusion, direct implantation of VX2 tumor fragments into the liver using a fine needle achieved a higher success rate than injecting VX2 tumor cells. The rabbit
\end{abstract}

Correspondence to: Professor Shu-Sen Zheng, Division of Hepatobiliary and Pancreatic Surgery, Department of Surgery, First Affiliated Hospital, School of Medicine, Zhejiang University, 79 Qingchun Road, Hangzhou, Zhejiang 310003, P.R. China E-mail: shusenzheng@zju.edu.cn

Key words: VX2 tumor, rabbit, liver neoplasm, angiography, computed tomography hepatic tumors inoculated by this method may therefore be a more suitable animal model for investigating liver cancer.

\section{Introduction}

Hepatocellular carcinoma (HCC) is one of the most common forms of malignant tumor occurring in the liver, and is the third leading cause of mortality from cancer worldwide $(1,2)$. Research has demonstrated that Southeast-Asian regions, including China, have a higher incidence rate of $\mathrm{HCC}$, due to the endemic nature of hepatitis $\mathrm{B}$ and $\mathrm{C}$ in these countries $(2,3)$. Surgical interventions, including complete resection and liver transplantation, demonstrate potentially curative treatments when the tumor is restricted to the liver, which offer the best prognosis for HCC $(4,5)$. However, surgical resection is only possible in a small proportion of patients with $\mathrm{HCC}$ due to advanced liver disease, extrahepatic metastases or underlying severe liver cirrhosis, inadequate functional hepatic reserve and poor general condition of the patient $(6,7)$. The difficulties posed by HCC treatment have prompted the development of a number of potential, non-surgical treatments, including transcatheter arterial chemoembolization (TACE), radiofrequency ablation (RFA), percutaneous ethanol injection therapy (PEI) and high-intensity focused ultrasound (HIFU) therapy (8-10). A suitable hepatic tumor animal model is crucial for the investigation of liver cancer diagnostics and therapeutics $(11,12)$.

VX2 carcinoma is an animal carcinoma, first developed by Shope and Hurst (13), and is derived from a rabbit papilloma. Previous research has demonstrated that the rabbit VX2 hepatic tumor grows rapidly, that its arterial blood supply is similar to that of human liver cancer and that its size is large enough to be observed by clinical imaging (14-15). As such, the rabbit VX2 hepatic tumor model has been widely used to study various aspects of liver tumor behavior and is generally accepted for the inoculation of liver cancer (16-18). However, in terms of the inoculation method, there are a number of different approaches to the induction of the VX2 tumor in the liver of recipient rabbits. In previous studies, VX2 tumor cell suspension was injected into the portal vein or hepatic artery (19), or directly into the liver parenchyma $(20,21)$ in order to achieve hepatic tumor growth. In addition, some investigators implanted small tumor pieces into the liver of 
rabbits by open laparotomy (22-24). Previous results have revealed varying degrees of success of tumor inoculation.

In the present study, in order to identify the preferred method for the inoculation of the VX2 tumor into the rabbit liver, we compared the injection of VX2 tumor cell suspension versus implantation of small, minced tumor fragment suspension using a fine needle. To the best of our knowledge, there is no literature comparing these two methods for inoculating VX2 tumors in the rabbit liver.

\section{Materials and methods}

Animals. This study was approved by the Animal Use and Care Committee of Zhejiang University (China). All New Zealand White rabbits used in this research, 2-3 months old, weighing $2-3 \mathrm{~kg}$, were provided by the experimental animal center, College of Medicine, Zhejiang University.

Preparation of suspension of VX2 cells and VX2 tumor fragments. Hind limb tumors of rabbits were used to propagate and maintain the VX2 tumor. All rabbits were anesthetized using a mixed solution of ketamine hydrochloride injection (25 mg/kg, intramuscular) and promethazine hydrochloride injection $(10 \mathrm{mg} / \mathrm{kg}$, intramuscular). With a fine needle, $0.2 \mathrm{ml}$ of VX2 cell suspension was injected into the muscles of the hind limbs of the rabbits. Following implantation into the hind limbs, the tumor grew rapidly. Two weeks following implantation, the tumor grew to a size of approximately $3 \mathrm{~cm}$ in diameter. Rabbits were later sacrificed and hind limb tumors harvested.

The VX2 tumor was aseptically resected from the hind limb and minced with surgical scissors and knives in normal saline (NS). The suspension was then filtered through an iron mesh with $0.08 \mathrm{~mm}^{2}$ pores to remove other tissue fragments. The filtrate was centrifuged at 1,500 rev/min for $8 \mathrm{~min}$ at room temperature and resuspended using NS at a concentration of $1 \times 10^{7}$ cells $/ \mathrm{ml}$.

After the tumor was stripped from the hind leg of the rabbit, the surrounding and necrotic tissues were removed from the tumor. The tumor was then cut into $1-\mathrm{mm}^{3}$ fragments and resuspended in a NS solution. The VX2 tumor fragment suspension was then ready for injection.

Implantation of VX2 tumors into liver. A total of 44 New Zealand White rabbits were randomly divided into 2 groups. All inoculations were performed by open laparotomy. To implant the VX2 tumor cell suspension or VX2 tumor fragments into the liver parenchyma, a sub-xyphoid laparotomy, approximately $3 \mathrm{~cm}$ in length, was performed to expose the left liver lobe following anaesthesia with the mixed solution of ketamine hydrochloride injection $(25 \mathrm{mg} / \mathrm{kg}$, intramuscular) and promethazine hydrochloride injection $(10 \mathrm{mg} / \mathrm{kg}$, intramuscular).

In group 1, a fresh VX2 tumor cell suspension containing $2 \times 10^{6}$ cells in a volume of $0.2 \mathrm{ml}$, was injected slowly into the liver parenchyma of the left lobe, using an 18-gauge needle with a 1-ml syringe. In group 2, following the exposure of the left lobe of the liver, a suspension containing $1-\mathrm{mm}^{3}$ fragments of VX2 tumor in a volume of $0.2 \mathrm{ml}$ was injected into the parenchyma of the left lobe of the liver, using an 18-gauge needle with a 1-ml syringe (Fig. 1). Following removal of the needles, all puncture places were gently compressed for $5 \mathrm{~min}$ in order to prevent leakage of cancer cells and bleeding from the liver parenchyma. Following confirmation of the absence of leakage of cancer cells and bleeding, the liver was repositioned back to its original abdominal space. The abdominal incision was then closed in layers. Antibiotic ointment was applied along the suture line.

Computed tomography (CT) examination and angiography. A total of 2 weeks following liver tumor implantation, all rabbits were anesthetized and placed into a helical CT scanner (General Electric Co, Milwaukee, WI, USA). Each animal underwent a conventional CT-scanning series, including plan scanning and enhancement scanning in order to detect tumor growth. Immediately following CT-scanning, surgical cutdown of the right femoral artery of the rabbits and insertion of a 2.7-F-diameter microcatheter (Terumo, Tokyo, Japan) were performed in order to gain access into the celiac trunk or common hepatic artery. Selective angiography was then carried out using a digital subtraction angiography (DSA) system (Allura Xper FD 20; Philips Medical Systems, Best, The Netherlands) to identify the tumor.

Necropsy and histopathology. Following completion of the CT scan and angiography, each rabbit was sacrificed and underwent a full necropsy to determine the tumor growth in the liver and extrahepatic metastases. All liver tumors were removed for pathological examination using hematoxylin and eosin staining.

Statistical analyses. Statistical analyses were carried out using SPSS software (ver. 11.0; SPSS Inc., Chicago, IL, USA). The Chi-square test was employed to determine significant statistical differences between the groups. However, when the sample size was too small to use the Chi-square test, Fisher's exact test was used instead. A P-value (two-tailed) $<0.05$ was considered to indicate statistically significantly differences.

\section{Results}

All rabbits (donors and recipients) successfully tolerated VX2 tumor inoculation. No mortality was observed at the time of implantation. All liver tumors were confirmed upon CT-scan and angiography, and were further confirmed by necropsy and pathological examination with hematoxylin and eosin staining.

In group 1, 8 rabbits displayed no evidence of liver tumor growth at 2 weeks following implantation. Liver tumors were grown in 14 out of 22 rabbits with an overall success rate of $63.6 \%$. In group 2, only 1 rabbit displayed no evidence of liver tumor growth. Successful liver tumor growth was achieved in 21 out of the 22 rabbits with an overall success rate of $95.5 \%$. Upon further sub-analysis, single nodular tumors in the liver were observed in 4 out of 14 rabbits in group 1 (28.6\%) and 14 out of 21 rabbits in group 2 (66.7\%) (Figs. 2-5). Multinodular tumors in the liver were grown in 10 out of 14 rabbits in group 1 (71.4\%) and in 7 out of 21 rabbits in group 2 (33.3\%) (Fig. 6). In addition, extrahepatic metastases were observed in 5 out of 14 rabbits in group $1(35.7 \%)$ and in 1 of 21 rabbits in group 2 (4.9\%) (Fig. 7). There were statistically significant differences between the 2 groups. The overall liver tumor growth rate of 


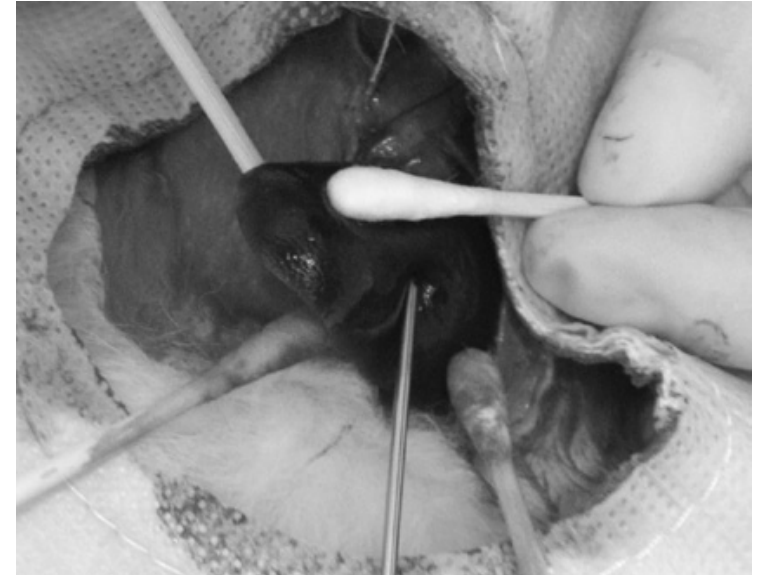

Figure 1. VX2 tumor fragment injection procedure. Using an 18-gauge needle with a 1-ml syringe, a suspension containing fragments of VX2 tumor in a volume of $0.2 \mathrm{ml}$ was injected slowly into the liver parenchyma of a rabbit in group 2 .

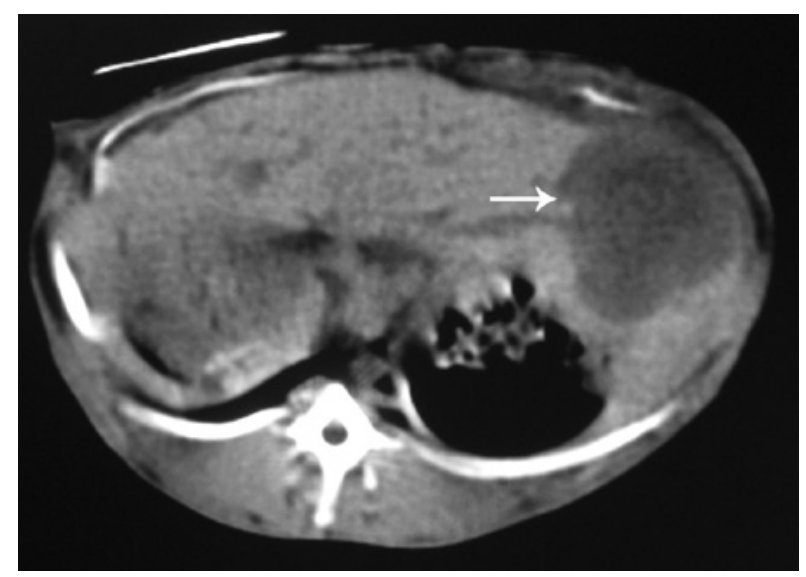

Figure 2. Abdominal $\mathrm{CT}$ image of a rabbit reveals a low-attenuated tumor in the left lobe of the liver (arrow). CT, computed tomography.

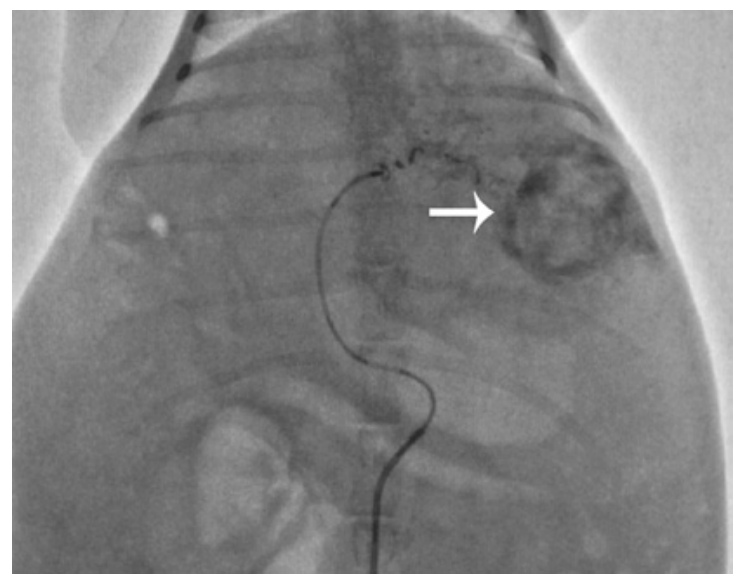

Figure 3. Hepatic angiography of the bearing cancer rabbit reveals a hypervascular tumor staining in the left lobe of liver (arrow).

group 1 was significantly lower than that of group $2(\mathrm{P}=0.025)$. Furthermore, the single nodular tumor growth rate of group 1 was significantly lower than that of group 2 , and the multi-

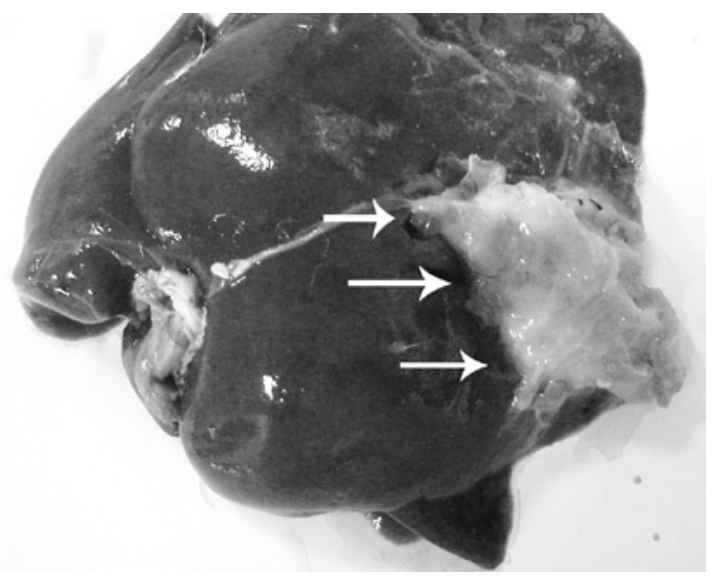

Figure 4. Gross image of a rabbit liver at 2 weeks following tumor fragment implantation. A solitary mass (arrow) grew in the left lobe of the rabbit liver.

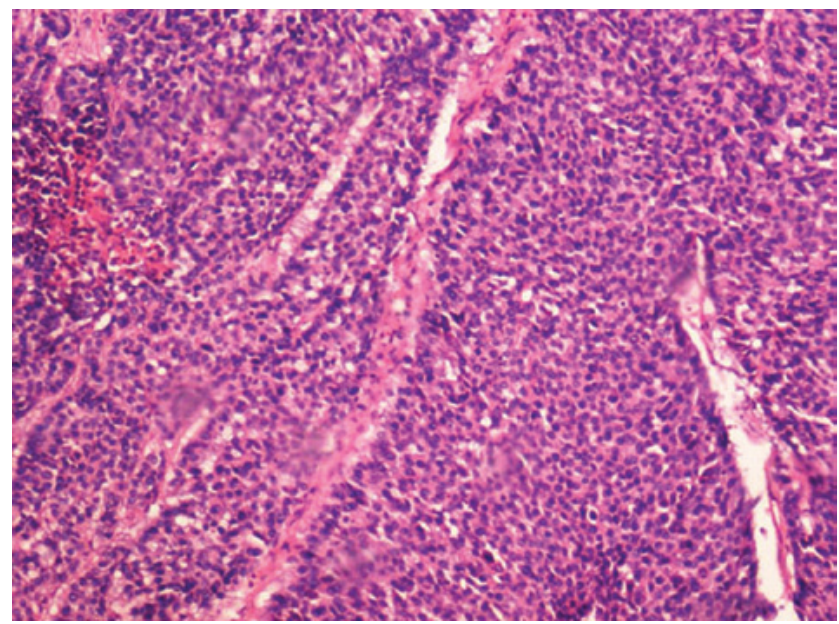

Figure 5. Histopathological image of the liver tumor of a rabbit (hematoxylin and eosin-stained, $\mathrm{x} 100$ ) reveals the viable VX2 tumor tissue. Typical cancer cells with malignant morphology are shown.

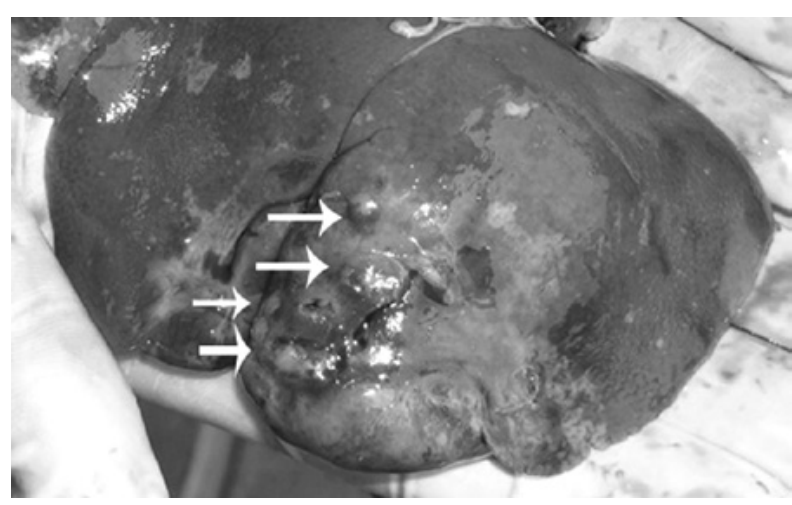

Figure 6. Gross image of a rabbit liver at 2 weeks following tumor cell implantation. Multiple small nodules were found over the surface of the liver (arrows) surrounding the incision site.

nodular tumor growth rate of group 1 was significantly higher than that of group $2(\mathrm{P}=0.041)$. Finally, a higher incidence of extrahepatic metastasis was observed in group $1(\mathrm{P}=0.028)$. 


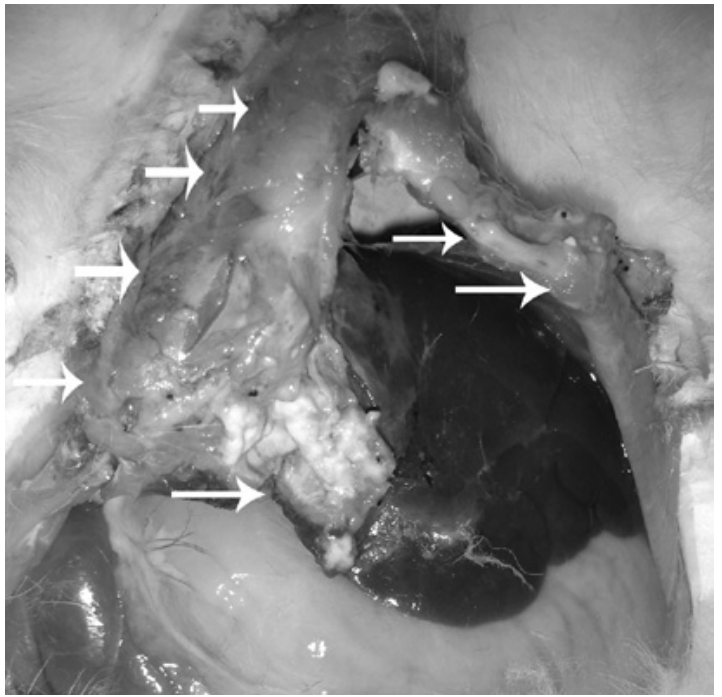

Figure 7. Gross image of a rabbit abdomen at 2 weeks following tumor cell implantation reveals a confluent tumor mass developed in the liver and abdominal wall (arrows).

\section{Discussion}

Liver cancer, particularly $\mathrm{HCC}$, is a form of highly malignant tumor with a poor prognosis of only several months survival from the time of diagnosis $(1,25)$. A suitable hepatic tumor animal model is essential for investigations of HCC diagnostics and therapeutics. Over the past few decades, despite the fact that VX2 carcinoma originated from rabbit malignant aplastic squamous cell carcinoma, it has been widely used as an experimental model for HCC studies of diagnosis and treatment due to its hepatic arterial supply and rapid growth pattern $(16,19-21)$.

In the present study, imaging and pathological examinations revealed that the VX2 liver tumor grew progressively 2 weeks following implantation. It is clear that successful tumor inoculation and growth in the rabbit liver leads to successful experimental investigations, and that incessant improvement of the tumor implantation technique may improve our knowledge regarding the selective use of the rabbit VX2 liver tumor. In addition, during previous similar experiments, we noticed that the implantation method of the tumor may not only affect the success rate of tumor growth and the animal expenses, but also, at times, the results of the study. Therefore, we initiated this study to attempt to identify a more desirable liver VX2 implantation method than previously.

Our experimental data revealed that the tumor growth success rate was as low as $63.6 \%$ in group 1, using the method of injecting VX2 cell suspension into the liver with a fine needle. Furthermore, multinodular liver tumor growth rates and extrahepatic metastasis rates were as high as 71.4 and $35.7 \%$, respectively. Tumor cells, injected into the liver, may not only enter the parenchyma, but also the bile ducts and liver blood vessels. Conversely, tumor cells may leak from the liver puncture site. These may cause the previously mentioned results of a lower tumor growth success rate, and a higher incidence of multinodular liver tumor growth and extrahepatic metastases. Despite ensuring that the needle was not in a bile duct or a vessel prior to injection of the tumor cells and compression of the puncture site in an attempt to achieve a better outcome, often the results remained unsatisfactory $(19,20)$. Conversely, the success rate of implanting liver VX2 tumors increased from 63.6 to $95.5 \%$ when a suspension of tumor fragments was used instead of the VX2 cell suspension. Moreover, the use of a VX2 tumor fragment suspension resulted in a higher liver single nodular tumor growth rate, as well as a statistically reduced incidence of extrahepatic metastasis.

Previously, certain investigators achieved a good liver tumor growth rate by implanting VX2 tumor fragments via surgical placement of these fragments in the liver parenchyma (26-29). Surgical implantation of VX2 tumor fragments controls the site of tumor growth more effectively, while tending to reduce the incidence of intra- and extrahaptic metastasis as compared to the method of injecting tumor cell suspension (28). However, there are also certain disadvantages of the surgical tumor fragment placement method. Of note, the surgical technique is more traumatic than the use of the cell suspension injection method using a fine needle. Surgical complications, including bile leakage, hemorrhage or abscess formation are more likely to occur (26). In addition, it is difficult to ensure that the implanted tumor fragment from the 'mother' tumor is viable tumor tissue. Unfortunately, failure of tumor induction may occur when necrotic tissue is placed into the liver. Our data revealed that the implantation of tumor fragment suspension into the liver directly using a fine needle (surgically placing VX2 tumor fragments into the rabbit liver) may be a more promising method than the inoculation method of injecting cell suspension (injecting tumor cells).

However, there were a number of limitations to the present study. Firstly, we did not compare all previously published induction methods of rabbit liver VX2 tumor. Secondly, the number of animals used in this research was small, which decreased the statistical power of the results, although this was sufficiently large to draw the main conclusion. Thirdly, the short-term follow-up period up of 2 weeks prevented the determination of the long-term fate of the hepatic tumor and the rabbits.

yIn conclusion, as a simple and effective method for the induction of a hepatic tumor, the direct implantation of VX2 tumor fragment suspension into the liver using a fine needle achieves a higher success tumor growth rate and a lower incidence of intra- and extrahepatic metastasis than the method of injecting VX2 tumor cells suspension into the liver using a fine needle. The results from our study may prove to be significant to future investigations and may help improve the efficiency of tumor inoculation, thereby reducing the number of rabbits required for study, as well as animal expenses.

\section{Acknowledgements}

This study was supported by the National Natural Science Foundation of China (No. 30901446), the Program for Innovative Research Team of Science and Technology of Zhejiang Province (No. 2009R50038), the Medical Health Fund of Zhejiang Province (No.2008A053), the Fundamental Research Funds for the Central Universities (2011KYJD023) and the Programme of Chinese Medical Science of Zhejiang Province (No.2009CB040). 


\section{References}

1. Parkin DM, Bray F, Ferlay J and Pisani P: Estimating the world cancer burden: Globocan 2000. Int J Cancer 94: 153-156, 2001.

2. He J, Gu D, Wu X, et al: Major causes of death among men and women in China. N Engl J Med 353: 1124-1134, 2005.

3. El-Serag HB: Hepatocellular carcinoma and hepatitis $\mathrm{C}$ in the United States. Hepatology 36: S74-S83, 2002.

4. Kawano Y, Sasaki A, Kai S, et al: Short- and long-term outcomes after hepatic resection for hepatocellular carcinoma with concomitant esophageal varices in patients with cirrhosis. Ann Surg Oncol 15: 1670-1676, 2008

5. Saab S, Yeganeh M, Nguyen K, et al: Recurrence of hepatocellular carcinoma and hepatitis B reinfection in hepatitis B surface antigen-positive patients after liver transplantation. Liver Transpl 15: 1525-1534, 2009.

6. Llovet JM, Fuster J and Bruix J: Prognosis of hepatocellular carcinoma. Hepatogastroenterology 49: 7-11, 2002.

7. Tsuzuki T, Sugioka A, Ueda M, Iida S, Kanai T, Yoshii H and Nakayasu K: Hepatic resection for hepatocellular carcinoma. Surgery 107: 511-520, 1990.

8. Scartozzi M, Baroni GS, Faloppi L, et al: Trans-arterial chemo-embolization (TACE), with either lipiodol (traditional TACE) or drug-eluting microspheres (precision TACE, pTACE) in the treatment of hepatocellular carcinoma: efficacy and safety results from a large mono-institutional analysis. J Exp Clin Cancer Res 29: 164, 2010.

9. Kurokohchi K, Masaki T, Watanabe S, et al: Time-lag performance of radiofrequency ablation after percutaneous ethanol injection for the treatment of hepatocellular carcinoma. Int J Oncol 28: 971-976, 2006.

10. Numata K, Fukuda H, Ohto M, et al: Evaluation of the therapeutic efficacy of high-intensity focused ultrasound ablation of hepatocellular carcinoma by three-dimensional sonography with a perflubutane-based contrast agent. Eur J Radiol 75: E67-E75, 2010.

11. Woodard LE, Keravala A, Jung WE, Wapinski OL, Yang Q, Felsher DW and Calos MP: Impact of hydrodynamic injection and phiC31 integrase on tumor latency in a mouse model of MYC-induced hepatocellular carcinoma. PLoS One 29: E11367, 2010.

12. Moroz P, Metcalf C and Gray BN: Histologic analysis of liver tissue following hepatic arterial infusion of ferromagnetic particles in a rabbit tumour model. Biometals 16: 455-464, 2003.

13. Shope RE and Hurst EW: Infectious papillomatosis of rabbits with note on histopathology. J Exp Med 58: 607-624, 1933.

14. Leander P, Golman K, Strande P, Klaveness J, Besjakov J and Fält K: A comparison between IEEC, a new biodegradable particulate contrast medium, and iohexol in a tumor model of computed tomography imaging of the liver. Invest Radiol 28: 513-519, 1993.

15. Merkle EM, Boll DT, Boaz T, et al: MRI-guided radiofrequency thermal ablation of implanted VX2 liver tumors in a rabbit model: demonstration of feasibility at $0.2 \mathrm{~T}$. Magn Reson Med 42: 141-149, 1999.
16. Sonoda A, Nitta N, Ohta S, et al: Controlled release and antitumor effect of pluronic F127 mixed with cisplatin in a rabbit model. Cardiovasc Intervent Radiol 33: 135-142, 2010.

17. Gupta T, Virmani S, Neidt TM, et al: MR tracking of iron-labeled glass radioembolization microspheres during transcatheter delivery to rabbit VX2 liver tumors: feasibility study. Radiology 249: 845-854, 2008.

18. Lee KH, Liapi E, Ventura VP, Buijs M, Vossen JA, Vali M and Geschwind JF: Evaluation of different calibrated spherical polyvinyl alcohol microspheres in transcatheter arterial chemoembolization: VX2 tumor model in rabbit liver. J Vasc Interv Radiol 19: 1065-1069, 2008.

19. Burgener FA and Violante MR: Comparison of hepatic VX2-carcinomas after intraarterial, intra-portal and intraparenchymal tumor cell injection: an angiographic and computed tomographic study in the rabbit. Invest Radiol 14: 410-414, 1979.

20. Izumi B, Tashiro S and Miyauchi Y: Anticancer effects of local administration of mitomycin $\mathrm{C}$ via the hepaticartery or portal vein on implantation and growth of VX2 cancer injected into rabbit liver. Cancer Res 46: 4167-4170, 1986.

21. Berkowitz DM, Alexander L and Hollenberg NK: a simple cell-suspension method for transplantation of VX2 carcinoma. J Natl Cancer Inst 54: 233-234, 1975.

22. Phillips JJ, Chang SL, Vargas HI, Dickman PS, Butler JA and Lipcamon JD: MR and CT imaging of ethanol-treated liver tumors in an animal model. Magn Reson Imaging 9: 201-204, 1991.

23. Ikeda Y, Matsumata T, Adachi E, Nishizaki T and Sugimachi K: Ethanol injection therapy in RBT-1 carcinoma of the rat liver evokes enhancement of metastasis. Surg Oncol 54: 9-12, 1993.

24. Thorstensen O, Isberg B, Svahn U, Jorulf H, Venizelos N and Jaremko G: Experimental tissue transplantation using a biopsy instrument and radiologic methods. Invest Radiol 29: 469-471, 1994.

25. Cabibbo G, Enea M, Attanasio M, Bruix J, Craxì A and Cammà C: A meta-analysis of survival rates of untreated patients in randomized clinical trials of hepatocellular carcinoma. Hepatology 51: 1274-1283, 2010.

26. Hänsler J, Neureiter D, Wasserburger M, et al: Percutaneous US-guided radiofrequency ablation with perfused needle applicators: improved survival with the VX2 tumor model in rabbits. Radiology 230: 169-174, 2004.

27. Yoon CJ, Chung JW, Park JH, Yoon YH, Lee JW, Jeong SY and Chung H: Transcatheter arterial chemoembolization with paclitaxel-lipiodol solution in rabbit VX2 liver tumor. Radiology 229: 126-131, 2003.

28. Nishizaki T, Matsumata T, Kanematsu T, Yasunaga $C$ and Sugimachi K: Surgical manipulation of VX2 carcinoma in the rabbit liver evokes enhancement of metastasis. J Surg Res 49: 92-97, 1990.

29. Virmani S, Rhee TK, Ryu RK, et al: Comparison of hypoxia-inducible factor-1alpha expression before and after transcatheter arterial embolization in rabbit VX2 liver tumors. J Vasc Interv Radiol 19: 1483-1489, 2008. 